\title{
Biodiversidade de Opiliones (Arachnida) em áreas de Cerrado e Mata Atlântica no Brasil
}

Os opiliões desempenham diferentes serviços ambientais, no entanto há poucas informações quanto a riqueza e distribuição dessas populações em diferente ecossistemas, mesmo em Unidades de Conservação (UC), como no estado de Minas Gerais, sudeste do Brasil. A partir disso, o objetivo do presente estudo visa analisar a diversidade de opiliofauna de duas Unidades de Conservação inseridas em ecossistemas distintos no estado de Minas Gerais. O Trabalho foi conduzido na Área de Proteção Ambiental do Rio Machado (APA), domínio de Mata Atlântica, e no Parque Nacional das Sempre Vivas (PARNA), área de Cerrado. As coletas foram, realizadas no período de setembro de 2018 a abril de 2019, com esforço amostral de 20 horas para o PARNA e 32 horas para a APA. Foi registrada uma nova espécie de Liogonyleptoides para a Ciência na APA Rio Machado, que possui maior riqueza que o PARNA. Não houve espécies em comum entre as duas áreas estudadas, reflexo de diferentes fatores associados, incluindo alta taxa de endemismo, o que justifica a conservação de ambas as Unidades de Conservação, pois alterações nesses ecossistemas poderiam acarretar a perda de espécies de opiliões para o estado de Minas Gerais.

\section{Biodiversity of Opiliones (Arachnida) in Cerrado and Atlantic Forest areas in Brazil}

The harvestmen perform different environmental services, however there is little information regarding the wealth and distribution of these populations in different ecosystems, even in Conservation Units (UC), as in the state of Minas Gerais, southeastern Brazil. From this, the objective of the present study aims to analyze the diversity of opiliofauna of two Conservation Units inserted in different ecosystems in the state of Minas Gerais. The work was carried out in the Environmental Protection Area of the Machado River (APA), Atlantic Forest domain, and in the National Park of Always Vivas (PARNA), Cerrado area. The collection were carried out from September 2018 to April 2019, with a sampling effort of 20 hours for PARNA and 32 hours for APA. A new species of Liogonyleptoides for Science was registered at APA Rio Machado, which has greater wealth than PARNA. There were no species in common between the two areas studied, reflecting different associated factors, including a high rate of endemism, which justifies the conservation of both Conservation Units, as changes in these ecosystems could result in the loss of species of harvestmen to the state of Minas Gerais.

Keywords: Liogonyleptoides; Discocyrtus invalidus; Gagrellinae.

Topic: Conservação da Biodiversidade

Reviewed anonymously in the process of blind peer.
Received: $10 / 03 / 2020$

Approved: 09/04/2020
Amanda da Silva Ferreira (iD

Instituto Federal do Sul de Minas Gerais, Brasil http://lattes.cnpq.br/1363418146951927

http://orcid.org/0000-0003-0809-4534

amanda1.ferreira@alunos.ifsuldeminas.edu.br

Isabella Luzia Cruvinel Pinheiro (it

Instituto Federal do Sul de Minas Gerais, Brasil

http://lattes.cnpq.br/5505266684431010

http://orcid.org/0000-0003-4256-9337

isaclpinh@gmail.com

Ludson Neves de Ázara (it)

Universidade Federal do Rio de Janeiro, Brasil

http://lattes.cnpq.br/4345253755018426

http://orcid.org/0000-0002-4826-3227

ludsonazara@yahoo.com.br

\author{
Mateus Aparecido Clemente (DD \\ Universidade Estadual Paulista Júlio de Mesquita Filho, Brasi \\ http://lattes.cnpq.br/6924671025219857 \\ http://orcid.org/0000-0003-4969-1335 \\ mateus1981@gmail.com \\ Marcos Magalhães de Souza \\ Instituto Federal do Sul de Minas Gerais, Brasi \\ http://lattes.cnpq.br/2334845279402555 \\ http://orcid.org/0000-0003-0415-1714 \\ marcos.souza@ifsuldeminas.edu.br
}

Referencing this:

FERREIRA, A. S.; PINHEIRO, I. L. C.; ÁZARA, L. N.; CLEMENTE, M. A.; SOUZA, M. M.. Biodiversidade de Opiliones (Arachnida) em áreas de Cerrado e Mata Atlântica no Brasil. Nature and Conservation, v.13, n.2, p.38-46, 2020. DOI: http://doi.org/10.6008/CBPC23182881.2020 .002 .0004 


\section{INTRODUÇÃO}

Opiliões são aracnídeos popularmente conhecidos como aranha-alho, viramundo, bodum, entre outros (ROCHA et al., 2007). Constituem a terceira ordem em diversidade da classe Arachnida, atrás apenas de ácaros e aranhas (SHEAR, 1982). A ordem engloba quatro subordens: Cyphophthalmi, Eupnoi, Dyspnoi e Laniatores (GIRIBET et al., 1999; MACHADO et al., 2007). Normalmente são onívoros, se alimentando de plantas ou animais, vivos ou mortos (KURY et al., 2002). São aracnídeos noturnos com hábitos crípticos, encontrados normalmente em lugares úmidos. Ocorrem em todos os ambientes terrestres, exceto na Antártida (HILLYARD et al., 1989).

Ecologicamente caracterizados por sua influência na reciclagem de matéria orgânica e ciclagem de nutrientes (MOORE et al., 1991), são um dos maiores indicadores de áreas com impactos de ação antrópica, tendo escassez de espécies em áreas fortemente antropizadas (DOCHERTY et al., 1997; KROMP et al., 1992; MEEK et al., 2002) e abundância em ecossistemas com nível elevado de conservação.

Estudos recentes vêm demonstrando efeitos negativos (perda de riqueza, e alteração na composição) da fragmentação de florestas, pastagem e queimadas florestais sobre as comunidades de opiliões (KROMP et al., 1992; DOCHERTY et al., 1997; MEEK et al., 2002; QUINN, 2004; TSUKAMOTO et al., 2005; CURRY et al., 1985; DENNIS et al., 2001), e ao uso de pesticidas utilizados em plantações (EPSTEIN et al., 2000), portanto podem ser bioindicadores para perturbações em florestas (KREMEN et al., 1993). Podem constituir modelos para estudos biogeográficos (GIRIBET et al., 2007), uma vez que sua baixa capacidade de colonização de novos ambientes torna a sua distribuição atual um reflexo de mudanças históricas, que acabaram por modificar o ambiente e isolar faunas, levando ao evento de especiação.

Portanto, somado a sua grande sensibilidade e pouca dispersão, são propensos a extinção (ROCHA, 1999). A atividade humana em função do crescimento das cidades e áreas de agricultura são responsáveis pela alteração na biodiversidade tropical (LAMBIN et al., 2003) e fragmentação florestal (LAURANCE et al., 2001; ALENCAR et al., 2004; LAURANCE, 2004). Para a conservação das espécies, a qualidade ambiental parece ser mais significativa do que o tamanho do fragmento (FLEISHMAN et al., 2002; SUMMERVILLE et al., 2004).

O Brasil possui cerca de 1008 espécies descritas (KURY, 2019), com destaque para o bioma Mata Atlântica, que abriga a maior diversidade do mundo (ROCHA, 1999). Por isso em estudos de inventários nesse ecossistema o número de espécies por localidade pode chegar a 50, o que reflete em altas taxas endêmicas (ROCHA et al., 2005). Esse padrão difere de regiões mais secas, como Caatinga ou Cerrado, onde esses animais são menos diversos, encontrando em média cerca de 10 espécies por estudo (BRAGAGNOLO et al., 2003).

Apesar dessa diversidade no país, há ecossistemas, Unidades de Conservação e estados sub amostrados, como Minas Gerais, que apresenta uma grande riqueza de biomas e áreas prioritárias para estudo e conservação de invertebrados. O Parque Nacional das Sempre-Vivas, no Vale do Jequitinhonha, e a região da Área de proteção ambiental da bacia hidrográfica do Rio Machado, no sul do estado, são exemplos 
de áreas prioritárias e que carecem de estudos (DRUMMOND et al., 2005).

Nesse estado há registro na literatura de quatro estudos que trazem lista de espécies (SOARES et al., 1970; SOARES, 1970; ÁZARA et al., 2016; FERREIRA et al., 2019). Considerando o exposto se espera que áreas de Mata Atlântica tenham maior riqueza de opiliones quando comparadas às áreas de Cerrado, e baixa similaridade de fauna. Na condição indicada, o presente estudo visa analisar a diversidade de opiliofauna de duas Unidades de Conservação inseridas em ecossistemas distintos no estado de Minas Gerais

\section{MATERIAIS E MÉTODOS}

O trabalho foi conduzido em duas Unidades de Conservação no estado de Minas Gerais (Figura 1): o

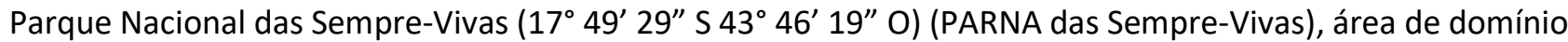
do bioma Cerrado, com fitofisionomias de campo sujo, sensu stricto, veredas, mata de galeria, campo rupestre e enclaves de mata, na região do Vale do Jequitinhonha. O clima é temperado úmido (KÖPPEN, 1936), esta Unidade de Conservação abrange os municípios de Bocaiúva, Buenópolis, Diamantina e Olhos-d' Água, com uma área de 124.154,47 hectares. O estudo foi realizado no período de outubro de 2018 a março de 2019, com 10 dias de coleta, no total de 20 horas de esforço de amostragem. A média de umidade relativa do ar do período de estudo foi de 82,24\%. Licença (SISBIO) 63930-1.

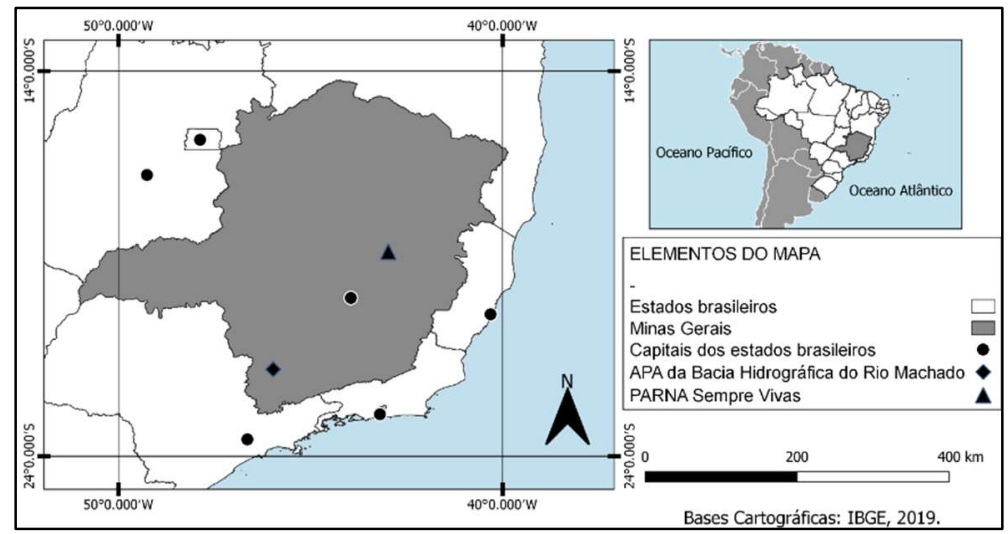

Figura 1: Localização do Parque Nacional (PARNA) das Sempre Vivas e da Área de Proteção ambiental (APA) da bacia hidrográfica do Rio Machado, estado de Minas Gerais, onde foram amostrados a opiliofauna (Arachnida, Opiliones).

A Área de Proteção Ambiental da Bacia Hidrográfica do Rio Machado (APA do Rio Machado) (214ㄴ' $\left.40.73^{\prime \prime} \mathrm{S} 45^{\circ} 58^{\prime} 31.65^{\prime \prime O}\right)$, com $1.016 \mathrm{~km}^{2}$, inclui áreas dos municípios Espírito Santo Dourado, Congonhal, Ipuiúna, São João da Mata, Poço Fundo, Carvalhópolis, Campestre, Machado, Alfenas, Paraguaçu e Fama, sul do estado de Minas Gerais. Esta APA possui o predomínio de floresta estacional semidecidual, fitofisionomia de domínio de Mata Atlântica, clima temperado úmido (KÖPPEN, 1936). O estudo foi realizado no período de outubro 2018 a abril de 2019, com 16 dias de coleta, no total de 32 horas de esforço de amostragem. A média de umidade relativa do ar do período de estudo foi de 82,46\%. Licença SISBIO 63914-1 e IEF/MG 062/2018.

O método utilizado para a coleta de opiliões foi a busca ativa noturna, considerada a mais eficiente dentre os métodos de captura desses animais (ROCHA et al., 2006). Dessa forma, com o auxílio de lanternas, procurou-se pelos animais em barrancos, troncos em decomposição, superfície de caules e folhas, cavidades 
rochosas e serapilheira. As coletas de adultos foram realizadas entre os horários de 19:00 a 21:00, período de maior atividade (BRAGAGNOLO et al., 2003), realizadas em pontos estratégicos delimitados com auxílio de funcionários do PARNA e da APA, onde existem ambientes com condições propícias para a ocorrência dos aracnídeos. Os opiliões coletados foram armazenados em álcool 70\% e enviados para o especialista Ludson Neves de Ázara na Universidade Federal do Rio de Janeiro (UFRJ), para identificação através de chaves dicotômicas e ficaram armazenados na coleção da instituição (Número de tombo MNRJ 59042 a 60613)

Foi calculado o índice de diversidade Shannon_H, equitabilidade e dominância utilizando o software PAST 2.1 (HAMMER et al., 2001). Para estimar o número de espécies das áreas foi utilizado estimadores jackknife 1, Chao 2 e Mao Tao, com o uso do programa Software R (R DEVELOPMENT CORE TEAM, 2017), com 1000 reamostragens. Este estimador é não paramétrico e tem como intuito indicar se os números de amostras foram suficientes para registrar o número total de espécies de um determinado local. Para verificar se houve diferenças significativas dos valores de diversidade, dominância e equitatividade entre as duas áreas estudadas (APA e PARNA) utilizou-se o teste de não paramétrico de Kruskal-Wallis (ZAR, 1999).

Para o cálculo de similaridade da fauna foi utilizada matriz binária, com dados de presença-ausência de espécies em cada região para análise de agrupamento, usando a matriz de similaridade obtida pela comunidade de Jaccard coefficient (S J). Todos os dados foram processados no software PAST 2.1 (HAMMER et al., 2001) e FITOPAC 2.1 (SHEPHERD, 2010). Foram comparadas as presentes áreas com outros estudos em áreas de influência do bioma Mata Atlântica (SOARES et al., 1970; SOARES, 1970; ROCHA et al., 2005; BRAGAGNOLO et al., 2003; FERREIRA et al., 2019; RESENDE et al., 2012a; 2012b), Mata Atlântica e Cerrado (ÁZARA et al., 2016); em Cerrado e Caatinga (SOUZA, 2014), estudo este que é um compilado de dados de diferentes estudos realizados na Caatinga.

\section{RESULTADOS}

Foram registradas sete espécies (quatro ao nível específico) para o PARNA das Sempre-Vivas (Cerrado), sendo Gagrellinae sp.1 (27) a espécie com maior abundância, seguida por Holoversia nigra (19) representando $75 \%$ dos indivíduos coletados. Para a APA do Rio Machado (Mata Atlântica) foram amostradas 11 espécies (oito ao nível específico) e Discocyrtus invalidus (84) foi a mais abundante, seguida por Gagrellinae sp. 2 (38) que juntas representaram 77\% dos indivíduos amostrados. Um dos morfótipos registrados para a APA do Rio Machado, Liogonyleptoides sp. 1 (Figura 2), compreende uma espécie não descrita para a ciência (Tabela 1).

Para a APA do Rio Machado o teste de jackknife de primeira ordem estimou 15,68 espécies, e neste estudo se registrou 11 espécies. Para a área PARNA das Sempre-Vivas foi estimado uma riqueza de 9,7 e registrou sete espécies neste estudo (Figura 3 A e B).

A análise de diversidade, dominância e equitatividade revelou que a APA do Rio Machado possui maior biodiversidade de opiliões (Tabela 2), no entanto os valores registrados não apresentaram diferenças significativas ((p) Kruskal-Wallis $=0.1056)$. 


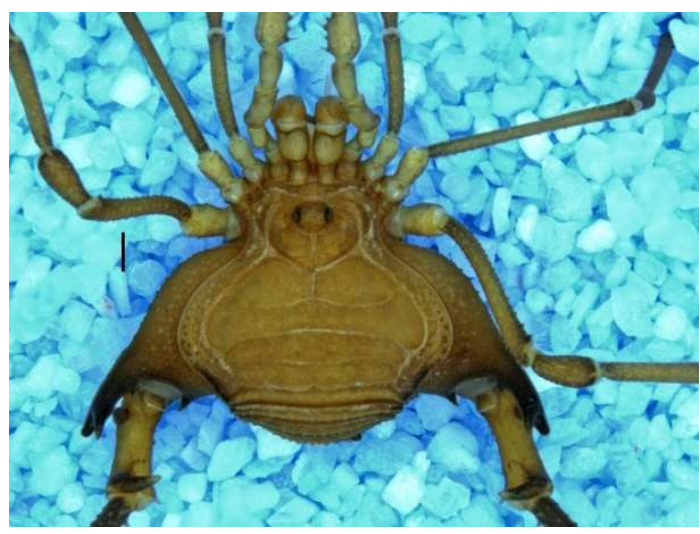

Figura 2: Novo registro para a ciência, Liogonyleptoides sp. 1, coletado na APA do Rio Machado.
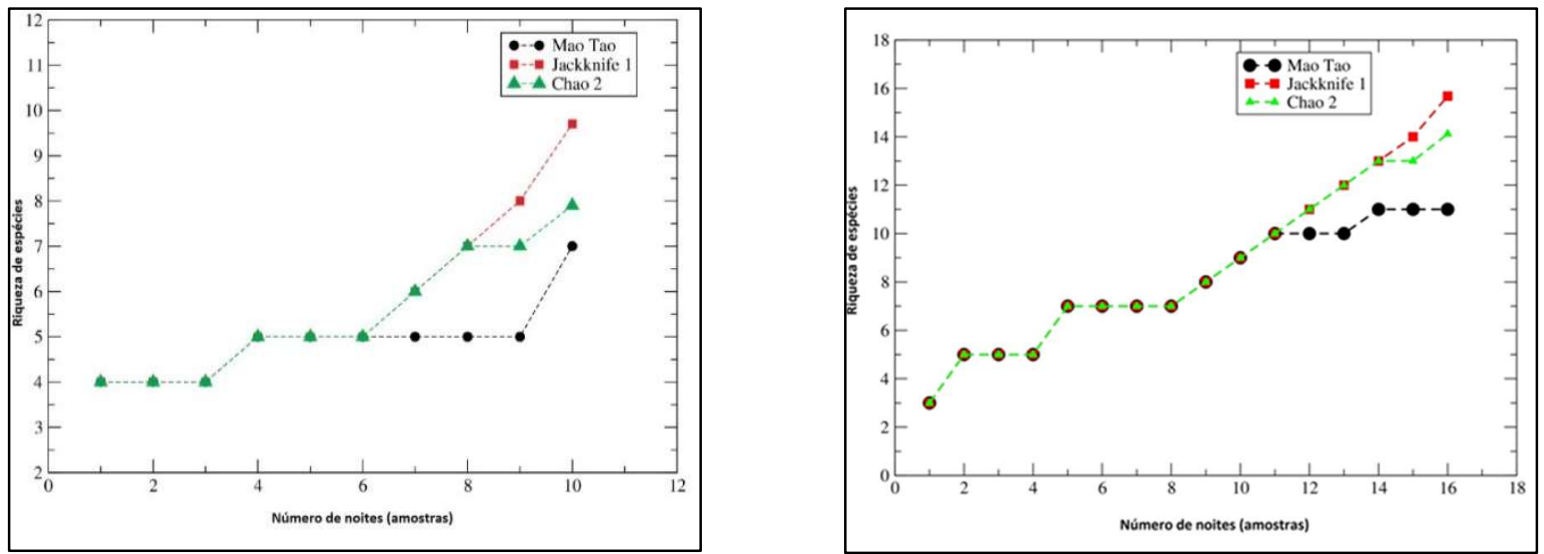

Figura 3: Figura 03 A- Número de espécies de opiliões estimadas (estimador jackknife 1, Chao 2 Mao Tao) para o

Parque Nacional das Sempre Vivas (PARNA das Sempre-Vivas). B- Número de espécies de opiliões estimadas (estimador jackknife 1 Chao 2 e Mao Tao) para A Área de Proteção Ambiental da Bacia Hidrográfica do Rio Machado (APA Machado).

Tabela 1: Número de espécies e morfoespécies de opiliones (Arachnida) registradas no Parque Nacional das Sempre Vivas e da Área de Proteção Ambiental da Bacia Hidrográfica do Rio Machado, Minas Gerais, Brasil.

\begin{tabular}{|c|c|c|c|}
\hline Famílias & Espécies e morfoespécies & PARNA Sempre Vivas & APA Rio Machado \\
\hline Gonyleptidae & Holoversia nigra Mello-Leitão, 1940 & 19 & 00 \\
\hline Gonyleptidae & Eugyndes sp. 1 & 05 & 00 \\
\hline Cosmetidae & Metavononoides guttulosus (Mello-Leitão, 1935) & 06 & 00 \\
\hline Gonyleptidae & Mitogoniella indistincta Mello-Leitão, 1936 & 01 & 00 \\
\hline Sclerosomatidae & Gagrellinae sp. 1 & 27 & 00 \\
\hline Gonyleptidae & Mitobatinae sp. 1 & 02 & 00 \\
\hline Gonyleptidae & Gonyleptidae sp. 1 & 01 & 00 \\
\hline Gonyleptidae & Gonyleptidae sp. 2 & 00 & 02 \\
\hline Gonyleptidae & Discocyrtus invalidus Piza, 1938 & 00 & 84 \\
\hline Gonyleptidae & Discocyrtus pertenuis Mello-Leitão, 1935 & 00 & 01 \\
\hline Gonyleptidae & Mischonyx cuspidatus (Roewer, 1913) & 00 & 17 \\
\hline Gonyleptidae & Liogonyleptoides sp. 1 & 00 & 06 \\
\hline Gonyleptidae & Discocyrtus flavigranulatus Soares, 1944 & 00 & 03 \\
\hline Sclerosomatidae & Gagrellinae sp. 2 & 00 & 38 \\
\hline Gonyleptidae & Eusarcus sp. 1 & 00 & 02 \\
\hline Gonyleptidae & Acutisoma longipes Roewer, 1913 & 00 & 02 \\
\hline Gonyleptidae & Longiperna trembao ROCHA et al., 2010 & 00 & 01 \\
\hline Cosmetidae & Metavononoides barbacenensis (Mello-Leitão, 1923) & 00 & 02 \\
\hline Riqueza & & 7 & 11 \\
\hline Abundância & & 61 & 158 \\
\hline
\end{tabular}

Tabela 2: Análise da opiliofauna (dominância, diversidade e equitabilidade) da Área de proteção ambiental (APA) da Bacia do Rio Machado, predomínio de Floresta Estacional Semidecidual de domínio do bioma Mata Atlântica; e Parque Nacional (PARNA) Sempre Vivas, área de domínio do bioma Cerrado, Minas Gerais, Brasil.

\begin{tabular}{|l|l|l|}
\hline Localidades & PARNA Sempre Vivas & APA Rio Machado \\
\hline Índices & 0,3109 & 0,3247 \\
\hline Dominance_D & 1,404 & 1,499 \\
\hline Shannon_H & 0,7215 & 0,625 \\
\hline Equitability_J & \\
\hline
\end{tabular}


O índice de Jaccard tem como premissa indicar a proporção de espécies compartilhadas entre duas amostras em relação ao total de espécies. Quando as duas áreas do presente estudo, foram comparadas entre si e com outros trabalhos em diferentes biomas, a similaridade foi baixa (menor que 0,5). Também se observou que não foi registrado espécies em comum entre as duas fitofisionomias deste estudo (PARNA das Sempre-Vivas e a APA do Rio Machado) (Tabela 3).

Tabela 3: Matriz de similaridade (Jaccard) de opiliones (Arachnida) entre diferentes estudos de Mata Atlântica e ecossistemas áridos (Cerrado e Caatinga).

\begin{tabular}{|c|c|c|c|c|c|c|c|c|c|c|c|}
\hline & APA & PARNA & DE SOUZA \& & SOARES \& & SOARES, 19 & PINTO-DA-A & BRAGAGND & FERREIRA E & F. Ipanema & RESENDE ef & DE ÁZARA 2 \\
\hline APA & 1,0000 & & & & & & & & & & \\
\hline PARNA & 0,0000 & 1,0000 & & & & & & & & & \\
\hline DE SOUZA et al 2014 & 0,0000 & 0,0000 & 1,0000 & & & & & & & & \\
\hline SOARES \& SOARES, 1970 & 0,0000 & 0,0000 & 0,0000 & 1,0000 & & & & & & & \\
\hline SOARES, 1970 & 0,0000 & 0,0000 & 0,0000 & 0,0536 & 1,0000 & & & & & & \\
\hline PINTO-DA-ROCHA et al 2005 & 0,0128 & 0,0000 & 0,0106 & 0,0339 & 0,0000 & 1,0000 & & & & & \\
\hline BRAGAGNOLO \& PINTO-DA-ROCHA 2 & 0,0000 & 0,0000 & 0,0000 & 0,0116 & 0,0000 & 0,0700 & 1,0000 & & & & \\
\hline FERREIRA et al 2019 & 0,1429 & 0,0909 & 0,0000 & 0,0172 & 0,0667 & 0,0260 & 0,0233 & 1,0000 & & & \\
\hline F. Ipanema e PECB RESENDE et al 201 & 0,0588 & 0,0000 & 0,0000 & 0,0128 & 0,0286 & 0,0313 & 0,0323 & 0,0286 & 1,0000 & & \\
\hline RESENDE et al 2012 & 0,0000 & 0,0000 & 0,0000 & 0,0000 & 0,0345 & 0,0109 & 0,0357 & 0,0000 & 0,5152 & 1,0000 & \\
\hline DE ÁZARA 2016 & 0,0556 & 0,0714 & 0,0606 & 0,0000 & 0,0000 & 0,0123 & 0,0000 & 0,1176 & 0,0000 & 0,0000 & 1,0000 \\
\hline
\end{tabular}

\section{DISCUSSÃO}

Todas as 10 espécies identificadas ao nível específico, amostradas para a APA do Rio Machado e para o PARNA das Sempre-Vivas compreendem novas ocorrências para estas unidades de conservação, ampliando a distribuição de suas populações. Uma das espécies, Holoversia nigra, havia sido registrada apenas para a sua localidade tipo, Serra do Cipó (Minas Gerais), área inserida no bioma Cerrado, similar ao PARNA das Sempre-Vivas.

As espécies Discocyrtus invalidus e Mischonyx cuspidatus são espécies com ampla distribuição (KURY, 2003; ÁZARA et al., 2018) e comumentemente encontradas em regiões perturbadas, sendo um indicativo da condição de espécies sinantrópicas. Apesar disto, os registros aqui para estas duas espécies compreendem novas ocorrências.

A espécie não descrita, Liogonyleptoides, pertence a subfamília Gonyleptinae, sendo seu gênero composto por cinco espécies conhecidas até o momento distribuídas no bioma Mata Atlântica: Liogonyleptoides capichaba Soares et al. (1946), no Espírito Santo; Liogonyleptoides minensis e Liogonyleptoides heliae, em Minas Gerais; Liogonyleptoides tetracanthus no Rio de Janeiro; e Liogonyleptoides inermis em Minas Gerais, Rio de Janeiro, Santa Catarina e São Paulo (KURY, 2003). Desta forma, este registro é um indicativo de como novas espécies podem ser encontradas em estudos de inventariamento faunístico.

A maior riqueza de espécies na APA pode ser reflexo de diferentes aspectos associados, como fitofisionomia, substratos de nidificação, endemismo, baixa mobilidade dos opiliões entre outros, e não unicamente dependente de um fator, a exemplo a umidade do ar, como já discutido para espécies bromelícolas que selecionam o local de oviposição por diferentes fatores bióticos e abióticos (OSSES et al, 2007). Essa condição de múltiplos fatores permite entender variação faunística entre as áreas, mesmo com 
valores de umidade do ar similares. Isso não descarta a relevância da umidade do ar para os opiliões, pois é importante na seleção de habitat, são pistas da qualidade do local, mas quando são mal selecionados, alguns habitats podem ser fisiologicamente estressantes, tendo influência negativa na sobrevivência dos indivíduos (STAMPS, 2009; MORIN, 2011). Opiliões possuem uma grande sensibilidade a perda de água e é comum se abrigarem em locais com baixa incidência luminosa e de alta umidade (SANTOS, 2003; SANTOS, 2007; REQUENA et al., 2012; BUZATTO et al., 2011). Em geral se escondem em cavidades naturais ou artificiais de rochas ou árvores, embaixo de troncos caídos (SANTOS, 2007), e repousam em folhas, seja na face abaxial ou adaxial de folhas em vegetação ciliar (BUZATTO et al., 2011), no entanto, esses aracnídeos, de acordo com o tipo de ambiente, toleram variações dos fatores abióticos (CURTIS et al., 2007).

No presente estudo verificou-se uma elevada taxa de endemismo para as espécies de opiliões, sendo um fator relevante, pois muitas espécies possuem uma distribuição geográfica restrita, como discutido por Rocha et al. (2005), resultado da baixa capacidade de deslocamento desses animais (SOUZA et al., 2014).

Tal fato se confirma quando verificamos o grau de similaridade da fauna de opiliões entre os diferentes estudos analisados, mostrando-se baixo (menor que 0,5 ). Na maioria dos casos não houve nenhuma espécie em comum (Tabela 3), mesmo em áreas de mesma fitofisionomia e geográficas próximas como o município de Poços de Caldas (SOARES et al., 1970), cerca de $40 \mathrm{~km}$ da APA do Rio Machado, o que reflete em uma fauna muito específica para cada localidade, como discutido por Souza et al. (2014).

Para o PARNA das Sempre-Vivas a similaridade de fauna foi ainda menor, não havendo nenhuma espécie em comum com $78 \%$ dos estudos comparados (Tabela 3). Apenas Mitogoniella indistincta foi registrada em comum com os estudos de Ázara (2016) e Ferreira et al. (2019). Tal fato se justifica, pois, a maioria dos trabalhos analisados foram realizados em domínio de Mata Atlântica, enquanto o PARNA está inserido em área de Cerrado. Tais fatores justificam a conservação de ambas as Unidades de Conservação, pois alterações nesses ecossistemas poderiam acarretar na perda de espécies de opiliões para o estado de Minas Gerais.

De acordo com os estimadores de espécies jackknife 1, Chao 2 e Mao Tao, a eficiência de coleta para o PARNA das Sempre-Vivas é de 58,3\%, já na APA Machado a eficiência registrada é de 73,3\%. Observou-se que para ambas as Unidades de Conservação não houve estabilização da curva de acúmulo de espécies, o que denota a necessidade de maior esforço amostral (Figura $2 \mathrm{~A}$ e B). Essa diferença pode ser resultado do maior número de dias de coleta na APA, e que também pode interferir na composição da fauna das áreas analisadas.

\section{CONCLUSÕES}

Áreas de domínio da Mata Atlântica apresentam maior riqueza de opiliões quando comparadas ao Cerrado, entretanto devido à ocorrência de espécies restritas e a diversidade similar, a conservação das Unidades de Conservação estudadas no presente trabalho são relevantes para assegurar a proteção da opiliofauna do estado de Minas Gerais. 
AGRADECIMENTOS: Ao SISBIO; ao IEF-MG; o Instituto Federal do Sul de Mina Gerais, Campus Inconfidentes; ao PARNA das Sempre Vivas.

\section{REFERÊNCIAS}

ALENCAR, A. A.; SOLÓRZANO, L. A.; NEPSTAD, D. C. Modeling forest understory fires in an eastern Amazonian landscape. Ecological Applications, v.14, n.4, p.139-149, 2004. DOI: https://doi.org/10.1890/01-6029

ÁZARA, L. N.; BERNARDI, L.; FERREIRA, R. L.. The first survey on harvestmen in Brazilian artificial cavities, with notes on distribution and natural history. Subterranean Biology, v.17, n.31, 2016.

ÁZARA, L. N.; FERREIRA, R. L.. Annotated checklist of Gonyleptoidea (Opiliones: Laniatores) associated with Brazilian caves. Zootaxa, v.4439, n.1, p.1-107, 2018. DOI: http://dx.doi.org/10.11646/zootaxa.4439.1.1

BRAGAGNOLO, C.; ROCHA, R. P.. Diversidade de opiliões do parque nacional da serra dos órgãos, Rio de Janeiro, Brasil (Arachnida: Opiliones). Biota Neotropica, v.3, n.1, p.1-20, 2003. DOI: https://doi.org/10.1590/S167606032003000100009

BUZATTO, B. A.; REQUENA, G. S.; LOURENÇO, R. S.; MUNGUÍA-STEYER, R.; MACHADO, G.. Conditional male dimorphism and alternative reproductive tactics in a Neotropical arachnid (Opiliones). Evolutionary Ecology, v.25, n.2, p.331-349, 2011.

CURRY, S. J.; HUMPHREYS, W. F; KOCH, L. E.; MAIN, B. Y. Changes in Arachnid Communities Resulting from Forestry Practices in Karri Forest, Southwest Western Australia. Australian Forest Research, v.15, n.4, p.469-580, 1985.

CURTIS, D. J.; MACHADO, G.. Ecology. In: ROCHA, R. P.; MACHADO, G.; GIRIBET, G.. Harvestmen: the biology of Opiliones. Massachussets: Harvard University Press, 2007. p.280-308.

DENNIS, P.; YOUNG, C. B.. The Effects of Varied Grazing Management on Epigeal Spiders, Harvestmen and Pseudoscorpions of Nardus Stricta Grassland in Upland Scotland. Agriculture, Ecosystems and Environment, v.86, n.1, p.39-57, 2001. DOI: https://doi.org/10.1016/S01678809(00)00263-2

DOCHERTY, M.; LEATHER, S. R.. Structure and abundance of arachnid communities in Scots and lodgepole pine plantations. Forest Ecology and Management, v.95 n.3, p.197-207, 1997. DOI: https://doi.org/10.1016/S03781127(97)00024-8

DRUMMOND, G. M.; MARTINS, C. S.; MACHADO, A. M.; SEBAIO, F. A.; ANTONINI, Y. O.. Biodiversidade em Minas Gerais: um atlas para sua conservação. Belo Horizonte: Fundação Biodiversitas, 2005.

EPSTEIN, P. R.. Is global warming harmful to health?. Scientific American, v.283, n.2, p.50-57, 2000.

FERREIRA, A. S.; PINHEIRO, I. L. C.; SOUZA, M. M.. Opiliones (Arachnida) in a mixed forest in southern Minas Gerais state Brazil. Journal of Entomology and Zoology Studies, v.7, n.5, p.666-671, 2019.

FLEISHMAN, E.; RAY, C.; SJÖGREN-GULVE, P.; BOGGS, C. L.; MURPHY, D. D.. Assessing the roles of patch quality, area, and isolation in predicting metapopulation dynamics. Conservation Biology, v.16, n.3, p.706-716, 2002.
DOI: https://doi.org/10.1046/j.1523-1739.2002.00539.x

GIRIBET, G.; RAMBLA, M.; CARRANZA, S.; BAGUÑÀ, J.; RIUTORT, M.; RIBERA, C.. Phylogeny of the arachnid order Opiliones (Arthropoda) inferred from a combined approach of complete $18 \mathrm{~S}$ and partial 28S ribosomal DNA sequences and morphology. Molecular Phylogenetics and Evolution, v.11, n.2, p.296-307, 1999. DOI:

https://doi.org/10.1006/mpev.1998.0583

GIRIBET, G; KURY, A. B.. Phylogeny and biogeography. In: Harvestmen: The Biology of Opiliones. Cambridge: Harvard University Press, 2007. p.62-87.

HAMMER, O.; HARPER, D. A. T.; RYAN, P. D.. PAST: Paleontological statistics software package for education and data analysis. Palaeontologia Electronica, v.4, n.1, p.1-9, 2001.

HILLYARD, P. D.; SANKEY, J. H. P.. Harvestmen: keys and notes for the identification of the species. London: Brill Archive, 1989.

KÖPPEN, W.. Das geographische System der Klimate. In: KÖPPEN, W.; GEIGER, R.. Handbuch der Klimatologie. Berlin: Gebrüder Bornträger, 1936. p.1-44.

KREMEN, C.; COLWELL, R. K.; ERWIN, T. L.; MURPHY, D. D.; NOSS, R. A.; SANJAYAN, M. A.. Terrestrial arthropod assemblages: their use in conservation planning. Conservation biology, v.7, n.4, p.796-808, 1993. DOI: https://doi.org/10.1046/j.1523-1739.1993.740796.x

KROMP, B; STEINBERGER, K. H.. Grassy field margins and arthropod diversity: a case study on ground beetles and spiders in eastern Austria (Coleoptera: Carabidae; Arachnida: Aranei, Opiliones). Agriculture, Ecosystems \& Environment, v.40, n.1-4, p.71-93, 1992. DOI: https://doi.org/10.1016/0167-8809(92)90085-P

KURY, A. B.. Annotated catalogue of the Laniatores of the New World (Arachnida, Opiliones). Revista Ibérica de Aracnología, Zaragoza, v.1, p.1-337, 2003.

KURY, A. B.. Checklist of validgenera of Opiliones of the World. Museu Nacional UFRJ website, 2019.

KURY, A. B.; NOGUEIRA, A. L. C.. Annotated Check List of Type Specimens of Arachnida in the Museu Nacional-Rio de Janeiro. Museu Nacional, 1999.

KURY, A. B.; ROCHA, R. P.. 4.4 Opiliones. Amazonian Arachnida and Myriapoda. Pensoft Publishers, p.345-362, 2002.

LAMBIN, E. F.; GEIST, H. J.; LEPERS, E.. Dynamics of land-use and land-cover change in tropical regions. Annual review of environment and resources, v.28, n.1, p.205-241, 2003. DOI: https://doi.org/10.1146/annurev.energy.28.050302.105459

LAURANCE, W. F.; COCHRANE, M. A.. Synergistic effects in fragmented landscapes. Conservation Biology, v.15, n.6, p.1488-1489, 2001.

MACHADO, G.; ROCHA, R. P.; GIRIBET, G.. What are harvestmen? In: Harvestmen: the biology of Opiliones. 2007. 
MEEK, B.; LOXTON D.; SPARKS T.; PYWELL R.; PICKETT H.; NOWAKOWSKI M.. The effect of arable field margin composition on invertebrate biodiversity. Biological Conservation. v.106, p.259-271, 2002. DOI: https://doi.org/10.1016/S0006-3207(01)00252-X

MOORE, J. C.; HUNT, H. W.; ELLIOT, E. T.. Ecosystem perspectives, soil organisms and herbivores. In: Microbial mediation of plant-herbivore interactions. New York: Wiley, 1991, p.105-140.

MORIN, X.; THUILLER, W.. Comparing niche-and processbased models to reduce prediction uncertainty in species range shifts under climate change. Ecology, v.90, n.5, p.1301-1313, 2009.DOI: https://doi.org/10.1890/08-0134.1

OSSES, F.; MARTINS, E. G.; MACHADO, G.. Oviposition site selection by the bromeliad-dweller harvestman Bourguyiahamata (Arachnida: Opiliones). Journal of Ethology, v.26, n.2, p.233-241, 2008. DOI: https://doi.org/10.1007/s10164-007-0054-z

ROCHA, R. P.. Opiliones. In: BRANDÃO, C. R. F.; CANCELLO, E. M.. Invertebrados Terrestres: Biodiversidade do Estado de São Paulo: Síntese do conhecimento ao final do século XX. São Paulo: FAPESP, 1999.

ROCHA, R. P.; BONALDO, A. B.. A structured inventory of harvestmen (Arachnida, Opiliones) at Juruti River plateau, State of Pará, Brazil. Revista Ibérica de Aracnologia, v.13, p.155-162, 2006.

ROCHA, R. P.; GIRIBET, G.. Taxonomy. In: Harvestmen: the biology of Opiliones. 2007.

ROCHA, R. P.; SILVA, M. B.; BRAGAGNOLO, C.. Faunistic similarity and historic biogeography of the harvestmen of southern and southeastern Atlantic Rain Forest of Brazil. The Journal of Arachnology, v.33, n.2, p.290-300, 2005. DOI: https://doi.org/10.1636/04-114.1

QUINN, N. W.. The presettlement hardwood forests and wildlife of Algonquin Provincial Park: a synthesis of historic evidence and recent research. The Forestry Chronicle, v.80, n.6, p.705-717, 2004. DOI:

https://doi.org/10.5558/tfc80705-6

R DEVELOPMENT CORE TEAM. R: a language and environment for statistical computing. 2017.

REQUENA, G. S.; BUZATTO, B. A.; MARTINS, E. G.; MACHADO, G.. Paternal care decreases foraging activity and body condition, but does not impose survival costs to caring males in a Neotropical arachnid. Plosone, v.7, n.10, 2012. DOI: https://doi.org/10.1371/journal.pone.0046701

RESENDE, L. P. A.; ROCHA, R. P.; BRAGAGNOLO, C.. The harvestmen fauna (Arachnida: opiliones) of the parque estadual Carlos Botelho, and the floresta nacional de ipanema, São Paulo, Brazil. Biota Neotropica, v.12, n.4, p.146-155, 2012. DOI: https://doi.org/10.1590/S167606032012000400016

RESENDE, L.; ROCHA, R. P.; BRAGAGNOLO, C.. Diversity of harvestmen (Arachnida, Opiliones) in Parque da Onça Parda, southeastern Brazil. Iheringia Série Zoologia, v.102, n.1 p.99-105, 2012.DOI: https://doi.org/10.1590/S007347212012000100014

SANTOS, F. H.. Ecophysiology. In: Harvestmen: The Biology of Opiliones. 2007.

SANTOS, G. C.; HOGAN, J. A.; WILLEMART, R. H.. Associative learning in a harvestman (Arachnida, Opiliones). Behavioural Processes, v.100, p.64-66, 2013. DOI: http://dx.doi.org/10.1016/j.beproc.2013.07.021

SHEAR W. A.. Opiliones. In: PARKER, S. P.. Synopsis and classification of living organisms. New York: McGraw-Hill. 1982.

SHEPHERD, G. J.. FITOPAC 2.1.2: Manual do usuário. Campinas: UNICAMP, 2010.

SOARES, A. M.; SOARES, E. M.. Opiliones de Itatiaia (Opiliones: Gonyleptidae, Phalangodidae). Revista Brasileira de Biologia, v.30, n.3, p.339-350, 1970.

SOARES, H. E. M. Gonyleptids from Pocos de Caldas, State of Minas Gerais, Brazil (Opiliones, Gonyleptidae). Rev. Brasil. Biol., p.211-216, 1970.

SOUZA, A. M.; SILVA, M. B.; CARVALHO, L. S.; OLIVEIRA, U Opiliões laniatores do semiárido. Artrópodes do semiárido: biodiversidade e conservação. Feira de Santana: Printmídia, 2014.

STAMPS, J. A.; KRISHNAN, V. V.; WILLITS, N. H.. How different types of natal experience affect habitat preference. The American Naturalist, v.174, n.5, p.623-630, 2009. DOI: https://doi.org/10.1086/644526

SUMMERVILLE, K. S.; CRIST, T. O.. Contrasting effects of habitat quantity and quality on moth communities in fragmented landscapes. Ecography, v.27, n.1, p.3-12, 2004. DOI: https://doi.org/10.1111/j.0906-7590.2004.03664.x

TSUKAMOTO, J.; SABANG, J.. Soil macro-fauna in an Acacia mangium plantation in comparison to that in a primary mixed dipterocarp forest in the lowlands of Sarawak, Malaysia. Pedobiologia, v.49, n.1, p.69-80, 2005.

ZAR, J. H.. Biostatistical analysis. 3 ed. Rio de Janeiro: Prentice-Hall do Brasil Ltda., 1996.

A CBPC - Companhia Brasileira de Produção Científica (CNPJ: 11.221.422/0001-03) detém os direitos materiais desta publicação. Os direitos referem-se à publicação do trabalho em qualquer parte do mundo, incluindo os direitos às renovações, expansões e disseminações da contribuição, bem como outros direitos subsidiários. Todos os trabalhos publicados eletronicamente poderão posteriormente ser publicados em coletâneas impressas sob coordenação da Sustenere Publishing, da Companhia Brasileira de Produção Científica e seus parceiros autorizados. Os (as) autores (as) preservam os direitos autorais, mas não têm permissão para a publicação da contribuição em outro meio, impresso ou digital, em português ou em tradução. 